\title{
Non-binary youth: Access to gender-affirming primary health care
}

\author{
Beth A. Clark, ${ }^{1}$ Jaimie F. Veale, ${ }^{2}$ Marrie Townsend, ${ }^{3}$ Hélène Frohard-Dourlent, ${ }^{2}$ \& Elizabeth M. Saewyc ${ }^{2}$ \\ ${ }^{1}$ Stigma and Resilience Among Vulnerable Youth Centre, School of Nursing, Faculty of Applied Science, University of British Columbia, Vancouver, \\ Canada. \\ ${ }^{2}$ School of Psychology, Te Whare Wananga o Waikato: The University of Waikato, Kirikiriroa: Hamilton, Aotearoa: New Zealand. \\ ${ }^{3}$ Trans Care BC, British Columbia Provincial Health Services Authority, Vancouver, British Columbia, Canada.
}

\section{DOI: 10.1080/15532739.2017.1394954}

\begin{abstract}
:
Background: Transgender (trans) youth who identify outside the gender binary are a growing subpopulation. In this article, we document differences in access to gender-affirming health care between binary and non-binary identified trans youth and explore ways of meeting the health needs of non-binary youth within primary care settings.

Methods: The Canadian Trans Youth Health Survey is a national online survey of trans youth, 14-25 years, conducted in 2013-2014. Among the 839 participants who responded to gender identity items in the survey, $41 \%$ identified as non-binary. We compared demographic, health outcome, and health care access responses between non-binary and binary (trans girls/women and trans boys/men) youth.

Results: Non-binary and binary youth were similar in most demographics, including age, geographic distribution, and ethnocultural backgrounds, however a larger proportion (82\%) of non-binary youth were assigned female at birth. Older non-binary youth (aged 19-25) were significantly more likely to forego needed healthcare than older binary youth; no significant differences were found between younger (14-18) non-binary and binary youth in foregoing healthcare. Overall, non-binary youth (13\%) were significantly less likely than binary youth $(52 \%)$ to access hormone therapy, but they were more likely than binary youth to report experiencing barriers to accessing hormone therapy when needed.

Conclusions: Non-binary trans youth in Canada report challenges in accessing needed gender-affirming healthcare. Primary care providers are well-situated to integrate a broad range of gender-affirming care services into practice in order to address the unique needs of nonbinary youth. Future research is warranted to explore experiences of non-binary youth related to barriers to care and to explore how services can be designed and delivered to better meet the needs of non-binary youth seeking gender-affirming primary care.
\end{abstract}

KEYWORDS: adolescent, health services accessibility, non-binary, primary health care, transgender persons

growing number of transgender (trans) youth in Canada are identifying beyond the gender binary, with genders such as genderfluid, genderqueer, and agender. Non-binary is used as an umbrella term for genders that do not fit within the binary system of girl/woman or boy/man; youth with non-binary genders may or may not also identify as trans (Frohard-Dourlent, Dobson, Clark, Duoll, \& Saewyc, 2016). Non-binary individuals typically experience gender in a way that does not always or ever align with the sex assigned to them at birth. For example, a person who is genderfluid may shift between genders, while someone who is genderqueer may experience gender in a way that is not part of the gender binary (Jones, 2016; Rainbow Health Ontario \& Speck, 2016).

Disparities in health outcomes and health care access are documented among trans populations (Barrett, 2016; Connolly, Zervos, Barone, Johnson, \& Joseph, 2016; Kenagy, 2005; OlsonKennedy et al., 2016; Sperber, Landers, \& Lawrence, 2005). Mistreatment in health care settings, outright refusal of care, postponement of care, and encounters with doctors who are unaware of the heath needs of trans individuals are all cited as barriers to care for both trans individuals and those who identify as gender nonconforming (Reisner, Bradford et al., 2015). The small amount of research that has emerged on health care access for non-binary adults suggests that this group encounters barriers to care (e.g., refusal to treat, avoidance) and health disparities (e.g., suicide attempt) at even higher rates than binary trans populations (Harrison, Grant, \& Herman, 2012).

Non-binary youth are increasingly visible in our communities and in health care settings, however little attention has been paid to the specific health care needs of this population and how this may correlate with disparities in health care access. Emerging understanding of non-binary genders in our society is apparent in the media and in current health care guidelines that explicitly address the needs of non-binary individuals (Coleman et al., 2012; Deutsch, 2016; LGBT Health Program, 2015; Scelfo, 2015). While there has been progress in terms of awareness, policy, and health care practice guidelines, it is important to further our understanding of how non-binary youth health outcomes and experiences accessing gender-affirming care may be unique, so that this population can be better served.

\section{Absence of non-binary youth in the literature}

Historically, sex and gender have been conceptualized as binary 
categories (female-male and girl/woman-boy/man, respectively) with the assumption that gender "naturally" follows from the sex that one is assigned at birth. As a result of these traditional understandings of gender and sex, the existence and experiences of non-binary people have been largely absent from the academic literature. This is true even within subfields that focus on trans people and gender identity, as trans experiences tend to be conceptualized through an understanding of transition as binary, from one gender or sex to "the other." Binary transitions may fit a linear narrative - social transition, then endocrine therapy, then gender-affirming surgery - that aligns with historical medical expectations of what it means to transition to "the other sex". Scholars across disciplines have criticized this binary conceptualization as it does not acknowledge the wide range of variations and diversity found in both sex and gender and thus has systematically erased the realities of both intersex bodies and nonbinary genders (Browne, 2004; Fausto-Sterling, 2000; Halberstam, 1998).

Scholarship about non-binary people is emerging (Austin, 2016; Beemyn, 2015b, 2015a; Nicolazzo, 2014; Rankin \& Beemyn, 2012; Richards et al., 2016), including work that does not focus on, but is inclusive of, youth with non-binary genders (Craig, Austin, Brennan, \& Alessi, 2015; Wolowic, Heston, Saewyc, Porta, \& Eisenberg, 2016). This literature often highlights necessity to take into account the complexity of sexualities and genders (Bosse \& Chiodo, 2016), but it remains scarce and it is limited almost exclusively to social science research. Clinical research continues focus primarily on binary experiences, with some notable exceptions (Richards et al., 2016). As OlsonKennedy et al. (2016) note, this scarcity can be partly explained by the lack of clinical tools and measures that include "adequate mechanism[s] to capture those youth who have nonbinary gender identities" (p. 176). The overall lack of academic and clinical research on non-binary youth is problematic as it results in little information on which to base best practices.

\section{Health care access}

Youth with non-binary genders may have health needs that differ from youth with binary genders. For example, a youth may require low dose hormones or temporary use of hormones in order to bring their body into alignment with their gender (Hastings, 2017). The current WPATH Standards of Care for the Health of Transsexual, Transgender, and Gender Non-conforming People (Coleman et al., 2012) acknowledge that there are "unique experiences that may transcend a male/female binary understanding of gender" (p. 9) and clearly state that non-binary individuals should have access to care necessary to alleviate gender dysphoria. Non-binary genders among children and youth are specifically discussed in reference to psychological and social interventions indicating that "mental health professionals should not impose a binary view of gender" (p. 16). These statements demonstrate growing professional awareness of the needs of youth with non-binary genders and establish that gender affirming medical care should be available to those with non-binary genders. These changes reflect the overall evolution in standards of care, which have become less prescriptive (i.e., focusing on how care providers can assist patients in reaching their goals) and broadened the range of care providers whose scope of practice can include providing gender affirming care (e.g. general practitioners, nurse practitioners, and nurses). While non-binary genders are acknowledged in standards of care, guidance around specific challenges experienced by this population or their unique health care needs is lacking. In a recent review of literature related to trans youth mental health Connolly et al. (2016) note that nonbinary youth are not included in this literature and that further work is needed to understand the experiences of these youth and inform health care practices for this population (see also FrohardDourlent, Dobson, Clark, Doull, \& Saewyc, 2016).

Empirical studies have documented challenges trans youth and adults experience when attempting to access gender affirming care. Lack of available trans-competent health care providers, discomfort with care providers, and costs have been identified as barriers to gender affirming care in multiple studies (Barrett, 2016; Bauer, Zong, Scheim, Hammond, \& Thind, 2015; Gridley et al., 2016; Huft, 2008; Kenagy, 2005; Sperber et al., 2005). Other barriers include geographic distance from competent care and outright denial of services by health care providers (Barrett, 2016; Kenagy, 2005; Sperber et al., 2005). Additionally, youth encounter specific challenges, such as lack of supportive family environment (e.g. homeless, in government care, families unsupportive of gender goals), legal barriers to care without parental consent, uncoordinated and inconsistent care provision, and limited and/or delayed access to pubertal suppression and hormone therapy (Gridley et al., 2016; Huft, 2008). The term youth can apply to a broad range of ages (e.g. 10-25), and given the dependence many younger youth have on their parents and caregivers related to accessing health care, age-based differences in health care access and health outcomes may be worthy of study.

\section{Health disparities}

Health disparities, particularly differences in mental health, are well documented among trans youth populations. Research highlights trans youth as having an increased risk of some forms of substance use, but studies do not differentiate between binary and non-binary youth (De Pedro, Gilreath, Jackson, \& Esqueda, 2017; Reisner, Greytak, Parsons, \& Ybarra, 2015). A review by Connolly et al. (2016) confirmed increased rates of depression, suicidality, self-harm, and eating disorders across multiple quantitative studies. Access to gender-affirming medical care and support for social transition were both connected with better mental health outcomes (Connolly et al., 2016; de Vries et al., 2014; Olson, Durwood, DeMeules, \& McLaughlin, 2016). These findings align with prior research documenting improved quality of life, life satisfaction, mental health, and self-esteem among trans youth with supportive parents (Simons, Schrager, Clark, Belzer, \& Olson, 2013; Travers et al., 2012).

\section{Purpose of investigation}

The health care needs and experiences of non-binary youth are rarely addressed in the literature, with most studies focusing on health services or outcomes for binary identified youth or trans youth populations as a whole. Numerous gender clinics serving trans youth have opened their doors in recent months and years, within a context of greater societal awareness of trans identities and research supporting medical intervention for trans youth. This article is grounded in a gender affirmative approach to trans health care, which supports depathologization of gender, validity of selfidentified non-binary and binary genders, and health care that 
supports individuals to live in the gender that is most comfortable to them (Hidalgo et al., 2013). As awareness of non-binary genders continues to grow and health care providers seek to offer services that meet the needs of non-binary youth, it is important to understand if they are experiencing unique barriers to care or related health disparities. No research to date has explored the challenges faced by non-binary youth seeking access to health care and how to address these issues within primary care settings. The purpose of this article is therefore to explore how the health outcomes and experiences of non-binary youth compared to those of trans girls/women and trans boys/men when accessing general primary or trans-specific primary health care needs.

\section{Method}

\section{Measures}

The Canadian Trans Youth Health Survey was an online health survey that was developed drawing on questions from existing population health surveys suitable for adolescent (e.g., British Columbia Adolescent Health Survey) and young adult (e.g., Canadian Community Health Survey) populations (Veale, Frohard-Dourlent, Dobson, \& Clark, 2015). Most of the survey was dedicated to demographics and typical health topics - such as exercise, sleep, nutrition, sex, mental health, substance use, and health care access. To account for developmental differences, two versions of the survey were designed (i.e., younger youth ages 1418 and older youth (ages 19-25); some questions were identical, allowing for reporting of aggregate data, while others are reported separately by age category. Age 14 was selected as the lower limit, as many Canadian provinces and territories allow youth as young as 14 to consent to their own health care. Youth-focused trans health care services in Canada include people through age 25, which was set as the upper age limit. Survey questions for which data are reported in this article are detailed in the Appendix.

At the time the Canadian Trans Youth Health Survey was developed, no single measure of gender identity had been validated as developmentally appropriate for youth in anonymous surveys in either English or French. Additionally, existing gender identity measures were not inclusive of non-binary genders. Five separate questions were included on the survey in order to elicit youth feedback on how to measure gender; these questions were drawn from existing surveys of adults, clinical measures, and oneoff studies of youth that had limited psychometric testing of measures (e.g., Conron, Scout, \& Austin, 2008; Scheim \& Bauer, 2015; Smith, Stewart, Poon, Peled, Saewyc, \& McCreary Centre Society, 2014).

\section{Recruitment, eligibility, and data collection}

Trans and genderqueer youth were recruited from across Canada, through clinical settings (e.g., children's hospital gender clinics), community groups (e.g., drop-in peer support groups), Facebook advertisements, study investigator and youth advisory group networks, email, and word of mouth. Youth aged 14 to 25 who self-identified as trans and/or genderqueer were eligible to participate, with separate health questions given to younger (1418) and older youth (19-25). The survey was administered in both official languages of Canada (English and French) and was available online from October 2013 through May 2014. This study was approved by the University of British Columbia, University of Winnipeg, and Dalhousie University research ethics boards.

\section{Participants}

Of the 923 youth who responded to the survey, 843 responded to one or more questions about their gender identity. Respondents were categorized as non-binary $(\mathrm{n}=344,41 \%)$, trans boy/man ( $=356,42 \%)$, or trans girl/woman $(\mathrm{n}=139,17 \%)$ primarily based on their responses to the gender identity question that was best suited to non-binary genders. Youth were deemed to be binary if they selected either I am transgender and identify as a girl or woman or I am transgender and I identify as a boy or man, and non-binary if they selected I am transgender and identify in some other way. Twenty-five participants selected the remaining response option, I am not transgender and two participants skipped this question but responded to other gender identity questions in the survey. Of these 27 participants, 23 reported a non-cisgender identity on another gender identity question on the survey, What is your gender identity? which offered more than ten possible response options as well as an other option with a free text box (see Veale et al., 2015); 21 of these responses were categorized as non-binary, one as a trans boy/man, and one as a trans girl/woman. The remaining four participants could not be categorized due to three giving a response further suggesting that they were not trans and one reporting their gender as "awesome."

Non-binary youth had an average age of 20 years, mostly reported White as their only ethnocultural background (74\%), and mostly spoke English at home. The demographics of non-binary youth in comparison with binary trans youth are presented in greater detail in the Results section. Youth did not report where they first learned about the study, so the percentage of youth recruited through clinical and the various non-clinical sites is unknown.

\section{Data analysis}

SPSS 22.0 was used to conduct statistical analyses. ANOVAs were used to compare means on continuous variables across the three gender categories, with Bonferroni post-hoc tests to assess differences between these specific groups. Percentages across the three groups were also compared via crosstabulations, Fisher's exact tests, and Pearson's $\chi 2$ tests. Logistic and ordinal analyses with binary/non-binary gender and birth-assigned sex predicting health and care access items were conducted using generalized linear models.

\section{Results}

\section{Demographic comparisons}

Overall, non-binary youth were demographically similar to binary youth. Their mean age of $20.03(S D=3.09)$ was similar to the trans boys/men $(M=19.54, S D=2.93)$ and younger than the trans girls/women $\left(M=20.91, S D=2.88 ; F_{2,836}=10.61, p<.001\right)$. A similar majority of non-binary youth spoke English at home (92.4\%) as binary youth $(89.5 \%)$, and they reported a similar prevalence of ethnocultural backgrounds: $74.4 \%$ White only, $8.8 \%$ Aboriginal, 5.0\% East Asian, and 3.2\% reported three or more ethnicities $\left(\chi^{2}(9)=9.30, p=.41\right)$. Non-binary youth were distributed across the provinces at similar levels to the binary youth: Atlantic Provinces (15.8\%), Québec (13.8\%), Ontario (29.6\%), Prairie Provinces (7.9\%), Alberta (12.9\%), and British 
Table 1 Comparisons between trans youth with and without a reported history of pregnancy involvement.

\begin{tabular}{|c|c|c|c|c|c|c|}
\hline & $\begin{array}{l}\text { Non- } \\
\text { binary }\end{array}$ & Binary & $n$ & $\begin{array}{l}\text { Binary/non- } \\
\text { binary: } \chi^{2}(1) \text {, } \\
\text { odds ratio }\end{array}$ & $\begin{array}{l}\text { Birth-assigned } \\
\text { sex: } \chi^{2}(1) \text {, odds } \\
\text { ratio }\end{array}$ & $\begin{array}{l}\text { Interaction: } \\
\chi^{2}(1)\end{array}$ \\
\hline General mental health [Mean $(S D)]$ & $0.84(0.75)$ & $1.02(0.80)$ & 641 & $7.10 * *, 0.63$ & $2.36,1.58$ & 0.71 \\
\hline Non-suicidal self-injury past year & $67.2 \%$ & $55.7 \%$ & 603 & $5.16^{*}, 1.62$ & $8.16^{* *}, 2.61$ & 0.42 \\
\hline Seriously considered suicide past year & $51.9 \%$ & $49.3 \%$ & 584 & $0.04,0.85$ & $0.87,0.72$ & 0.76 \\
\hline Attempted suicide past year & $23.6 \%$ & $24.5 \%$ & 383 & $0.99,0.77$ & $0.06,1.13$ & 1.13 \\
\hline Drank alcohol weekly in the past month & $28.3 \%$ & $21.7 \%$ & 687 & $2.27,1.36$ & $0.01,0.97$ & 0.11 \\
\hline Used marijuana past year & $48.2 \%$ & $41.3 \%$ & 681 & $0.05,1.04$ & $3.37,0.56$ & $6.50^{*}$ \\
\hline Smoked a cigarette past month & $30.1 \%$ & $21.0 \%$ & 643 & $0.51,0.86$ & $2.61,1.76$ & $10.22 * *$ \\
\hline
\end{tabular}

$* p<.05 ; * * p<.01$; comparison groups for odds ratios were binary youth and assigned male at birth.

Columbia $(19.9 \%), \chi^{2}(5)=5.72, p=.34$. One notable and statistically significant difference was that a larger proportion of the non-binary youth had been assigned female at birth $(81.6 \%)$, compared to the distribution among the binary youth $(69.9 \%)$, $\chi^{2}(1)=14.26, p<.001$. As a result, sex assigned at birth is included in all further analyses to control for this.

\section{Health outcomes}

Mental health and substance use comparisons between binary and non-binary youth are given in Table 1 . An ordinal regression analysis was conducted for self-rated general mental health and this showed that after controlling for sex assigned at birth, for each one-unit increase on the response scale (e.g., going from Poor to Fair) non-binary youth were predicated to have a significantly $(37 \%)$ smaller odds of being at the at the higher mental health response option (see also Figure 1 for percentages of responses to this question).

Binary logistic regression analyses were conducted for the remaining health outcome variables which are outlined in Table 1. Non-binary youth had a $62 \%$ increased odds of reporting nonsuicidal self-injury in the past year, even after taking into account the significant difference for birth-assigned sex for this variable (Table 1). Non-binary youth were more likely to report weekly alcohol use (Fisher's exact test, $p=.05$ ), but this difference was not statistically significant in the logistic regression model once sex-assigned at birth was accounted for. There were no main effects for binary versus non-binary genders for marijuana and cigarette use, but there were significant interaction effects between

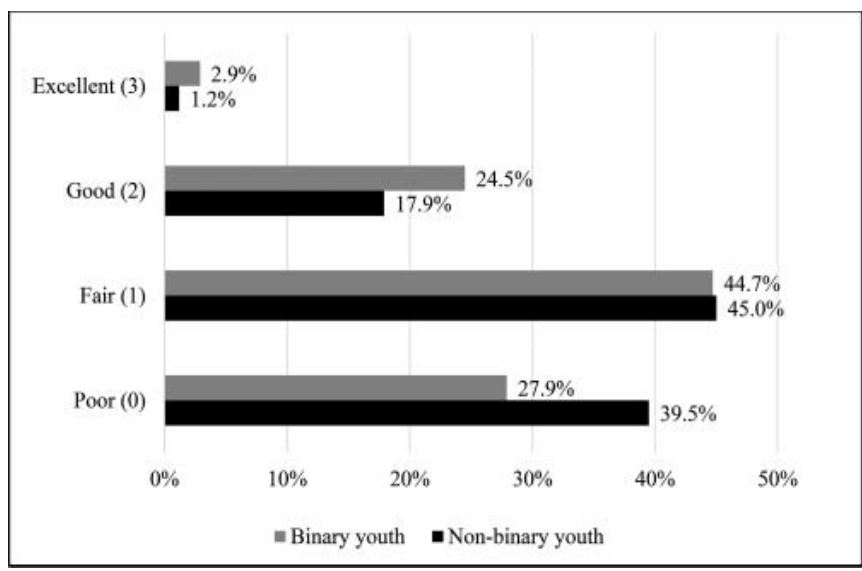

Figure 1 Self-reported mental health (scale 0 to 3 ) binary/non-binary genders and sex assigned at birth: non-binary participants assigned male at birth (AMAB) reported the highest rate of marijuana use $(59.6 \%, n=52)$, followed by non-binary participants assigned female at birth (AFAB) $(45.7 \%, n=232)$, trans boys/men $(43.9 \%, n=289)$, and trans girls/women $(34.5 \%$, $n=113$ ). Post-hoc comparisons were conducted between these groups which showed that non-binary AMAB participants had a significantly higher rate than trans girls/women (Fisher's exact test, $p<.01) .{ }^{1}$ Similarly, non-binary participants AMAB were most likely to report smoking in the past month $(43.9 \%, n=42)$, followed by non-binary participants AFAB $(28.1 \%, n=224)$, trans boys/men $(24.5 \%, n=273)$, and trans girls/women $(12.0 \%, n=$ 108). Post-hoc analyses revealed that trans girls/women scored significantly lower than all three other groups (all Fisher's exact test, $p<.01){ }^{1}$

\section{Health care access}

\section{Family doctors}

Logistic regression analyses outlined in Table 2 show that nonbinary participants had smaller odds of having a family doctor and had a much smaller odds of their family doctor knowing about their trans identity or experience. On a scale of 0 (very uncomfortable) to 3 (very comfortable), non-binary youth reported feeling less comfortable speaking with new doctors and family doctors about their trans status and trans-specific health care needs (Table 2). Figure 2 illustrates how participants in these gender groups responded regarding comfort with their family doctor.

\section{Foregone care}

Younger (14-18 year old) and older (19-25 year old) youth were asked different questions about whether or not they had foregone needed health care at any point in the previous 12 months. As outlined in the logistic regression models in Table 2, there were no significant differences in the rates of foregone physical or mental health care between non-binary and binary younger youth. Non-binary 19- to 25-year olds, however, were predicted to have almost double the odds of reporting had foregone needed healthcare in the previous 12 months.

\section{Hormone therapy}

Trans-specific health care was addressed through a series of questions about hormone therapy, including whether youth had ever taken hormones for trans-related reasons, and if yes, from what sources had they ever received their hormones. As outlined in Table 2, non-binary youth had smaller odds of reporting ever 
Table 2 Results of regression analyses for health care access.

\begin{tabular}{|c|c|c|c|c|c|c|}
\hline & $\begin{array}{l}\text { Non- } \\
\text { binary }\end{array}$ & Binary & $n$ & $\begin{array}{l}\text { Binary/non- } \\
\text { binary: } \chi 2(1) \text {, } \\
\text { odds ratio }\end{array}$ & $\begin{array}{l}\text { Birth-assigned } \\
\text { sex: } \chi 2(1), \text { odds } \\
\text { ratio }\end{array}$ & $\begin{array}{l}\text { Interaction: } \\
\chi 2(1)\end{array}$ \\
\hline Have a family doctor & $62.5 \%$ & $76.2 \%$ & 730 & $14.05^{* *}, 0.49$ & $0.73,1.30$ & 0.13 \\
\hline Family doctor knows transgender & $26.4 \%$ & $68.0 \%$ & 506 & $59.08 * *, 0.17$ & $0.01,0.97$ & 0.00 \\
\hline Comfort with a new doctor, $\mathrm{M}$ (SD) & $0.72(0.80)$ & $1.03(0.84)$ & 733 & $3.96 * *, 0.57$ & $3.96^{*}, 1.75$ & 2.56 \\
\hline Foregone physical health care (age 14-18) & $36.9 \%$ & $31.2 \%$ & 225 & $0.78,1.31$ & $0.06,1.18$ & 0.11 \\
\hline Foregone mental health care (age 14-18) & $73.8 \%$ & $65.4 \%$ & 228 & $0.78,1.33$ & $0.14,1.28$ & 0.32 \\
\hline Foregone health care (age 19-25) & $58.1 \%$ & $40.2 \%$ & 399 & $7.94 * *, 1.97$ & $0.03,1.07$ & 0.01 \\
\hline Ever taken hormone therapy & $12.8 \%$ & $49.8 \%$ & 700 & $68.07 * *, 0.39$ & $5.68^{*}, 0.39$ & 1.44 \\
\hline if so, taken non-prescription hormones & $37.1 \%$ & $21.3 \%$ & 220 & $3.32,2.38$ & $0.17,1.40$ & 0.44 \\
\hline
\end{tabular}

$* p<.05 ; * *<.01$; comparison groups for odds ratios were binary youth and assigned male at birth.

having taken hormones. Non-binary youth who had taken hormones were more likely to have ever received hormones from a non-prescription source (Fisher's exact test, $p=.02$ ), although this difference did not reach statistical significance in the logistic regression model after taking into account sex assigned at birth.

Participants were asked if they were currently taking hormones and those who were not taking hormones were asked why this was the case (Figure 3). Overall, the majority of participants indicated that hormone therapy was currently necessary for them, as they were either on hormones (36\%), in the process of getting started $(9 \%)$, or experiencing a barrier to care $(16 \%)$. The remaining youth were either undecided $(26 \%)$ or not planning on taking hormones $(13 \%)$. The differences between non-binary and binary youth responses to questions regarding hormone therapy status are presented in Figure 3. Non-binary youth were more likely to be still deciding about hormones or not planning on taking hormones, and less likely to be on hormones, in the process of starting, or experiencing a barrier to care $(\chi 2(4)=256.49, p<.001)$.

Among youth who needed hormone therapy, a significantly higher percentage of non-binary youth reported experiencing barriers to accessing hormones than binary youth. While this

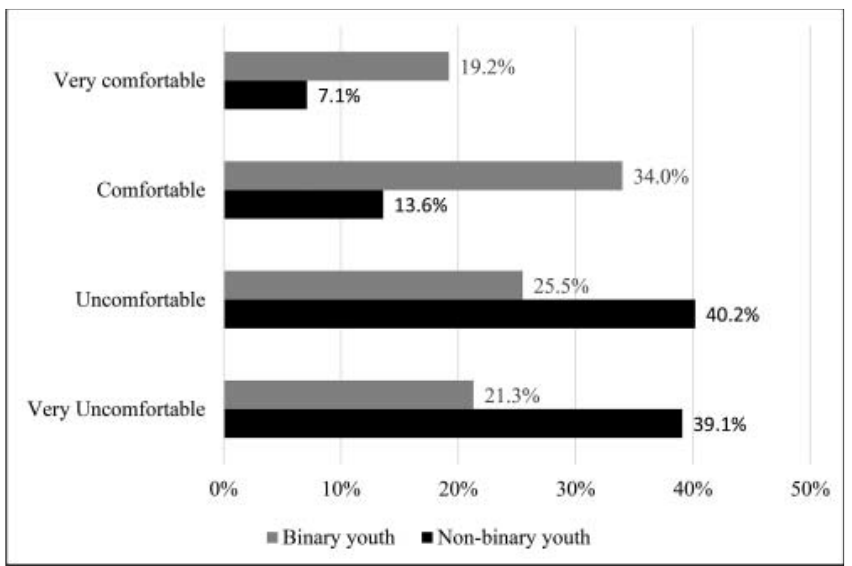

Figure 2 Comfort discussing trans-specific health care needs with family doctor difference did not reach statistical significance in the logistic regression model in Table 2, we ran an additional model without the interaction predictor and in this case having a non-binary gender was a significant predictor, Wald $\chi^{2}(1)=6.17, p=.013$, $O R=2.01$. In other words, the model predicted that non-binary youth twice the odds of experiencing a barrier to accessing necessary hormone therapy compared to binary youth.

The barrier to care most frequently identified by both nonbinary and binary youth was being unable to find a doctor to prescribe hormones. Lack of support from family was a barrier for multiple youth. One non-binary youth described their situation in this way, "I'm genderfluid. Although I've considered [hormone therapy], my dad would never allow it". Responses from nonbinary youth such as, "don't have money/support from family/friends", indicated they were facing multiple barriers to care. Other barriers described by non-binary youth included sports regulations, being pregnant, and lacking the health information they needed to make an informed decision about starting hormone therapy.

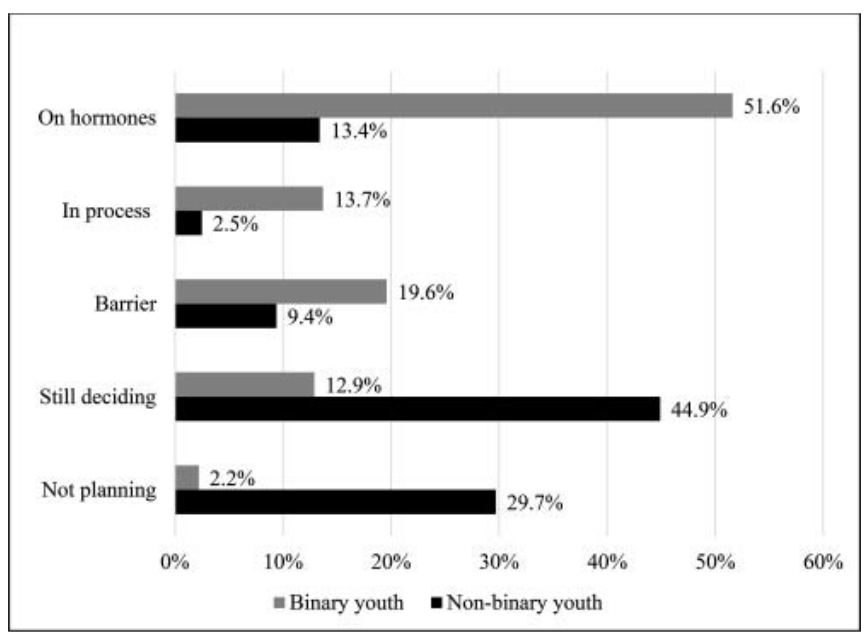

Figure 3 Hormone therapy status 


\section{Discussion}

Non-binary youth represent a growing and increasingly visible population with unique health care experiences and needs. This investigation is a first step in comparing health care access and outcome data between non-binary and binary trans youth. The results of our Canadian survey affirm several findings from the extant trans health literature: many trans youth lack access to needed gender-affirming care and experience discomfort in discussing their trans health care needs with primary care providers (Barrett, 2016; Bauer et al., 2015; Gridley et al., 2016; Reisner et al., 2015). Non-binary youth experienced these barriers to care at even higher rates than binary trans youth. In particular, non-binary youth were both less likely to have a family doctor and, when they had one, that doctor was less likely to know about their trans identity or experience.

Non-binary youth were less likely to require hormone therapy, but more likely to experience barriers to care when hormone therapy was needed. The reasons behind these findings are likely multi-faceted. With regard to the need for hormone therapy, more non-binary youth may live comfortably in their gender without the need for this care (e.g., needs met through other kinds of gender affirmation, such as pronoun use, names, clothing, hairstyles). The unavailability of this care in a youth's geographic area or lack of family support may foreclose consideration of hormone therapy as an option. High rates of indecision about hormone therapy could indicate a barrier to support around decision making or to information about the range of options that exist for hormone therapy (e.g., low-dose and temporary hormone use).

As indicated in previous research, barriers to care can take several forms, including primary care provider refusal to provide care or referrals, long waits for care, inability to travel to appointments, and financial barriers for mental health assessments and prescriptions (Barrett, 2016; Gridley et al., 2016; Huft, 2008; Sperber et al., 2005). Discomfort with primary care providers can in itself present a barrier to care. Many barriers may be exacerbated by societal stigma manifesting in encounters with primary care providers who dismiss the experiences of non-binary youth, rather than viewing their genders as valid and respecting their need to access gender-affirmative care. Youth in this survey reported additional barriers including pregnancy, sports regulations, and lack of information necessary for decision making.

The importance of parental support in trans youth's wellbeing has been well documented (Simons et al., 2013; Travers et al., 2012), however our analyses did not include the role that parent support may play in mediating access to gender-affirming care. Parental barriers to care can include refusing to provide various supports, such as permission to take hormones, transportation to appointments, or payment for prescriptions. The extent to which varying levels of parental support facilitate or impede access to needed gender affirming care, and how a lack of understanding and acceptance of non-binary genders may influence parental support, are worthy of further investigation.

Consistent with previous findings that trans youth experience better overall mental health with access to gender affirming medical care (Connolly et al., 2016; de Vries et al., 2014), nonbinary youth in our study reported not only higher barriers to needed care, but also significantly lower rates of overall mental health and higher rates of non-suicidal self-harm, even after controlling for the higher levels of non-suicidal self-injury among all participants AFAB in this study. Non-binary participants AMAB reported higher levels of marijuana and cigarette use than trans girls/women. As awareness and validation of non-binary genders continue to grow, it will be important to monitor changes in mental health and substance use rates, parental support, and access to gender-affirming health care, to identify interventions to reduce the health disparities faced by non-binary youth.

Overall, our findings point to the need to ensure that non-binary youth can consistently access gender-affirmative primary care in safer spaces, where their genders are viewed as valid and nonpathological, and standards of care are equivalent for binary and non-binary youth (Coleman et al., 2012; Hidalgo et al., 2013). Primary care providers are well-positioned to support non-binary youth who are seeking gender-affirming care because they practice in settings that are family-centered (e.g., family practice), youth-centered (e.g., youth clinic, school-based clinic), and close to home. Increased access to gender-affirming care in safer spaces could have a positive impact on rates of foregone physical or mental health care among older non-binary youth, as reluctance to access health care services may relate to having experienced, or fearing, unsupportive encounters (Kosenko, Rintamaki, Raney, \& Maness, 2013).

Health care providers themselves experience barriers to provision of gender-affirming care, in particular lack of training (Gridley et al., 2016; Poteat, German, \& Kerrigan, 2013; Vance, Halpern-Felsher, \& Rosenthal, 2015). Increasing primary care provider access to trans health education may serve to both increase access to care and address the reported discomfort nonbinary youth experience when discussing trans specific health care needs with their family doctors. Health care providers may be well served by formal education that addresses a broad range of needs (e.g., medical transition care, psychosocial needs, and family support), alongside education about how societal stigma shapes both the daily lives and the health care experiences of non-binary youth. However, immediate steps can be taken by primary health care providers to ensure their clinics are spaces that are safe enough for non-binary youth to build the trust necessary for open, honest dialogue about health care. This includes using language that is neutral, avoiding words that imply a binary approach (e.g., use of "another gender" instead of "opposite gender"), affirming that non-binary and binary genders are equally valid, using forms that do not make binary assumptions, refraining from making assumptions about what medical interventions a non-binary youth will or will not require, providing information about the full range of hormone therapy options available, and making resources available that include representation of non-binary genders.

\section{Future Research}

Given the relative size of the non-binary youth population ( $41 \%$ in our sample), along with the disparities noted in health outcomes and health care access, further exploration of how health care services can be designed and delivered to better meet the needs of non-binary youth is warranted. This can be achieved through studies specifically focused on non-binary youth and through research such as ours, which examines non-binary youth experiences within the context of the larger trans youth population. Additional quantitative research into health outcomes and risks 
and protective factors would be beneficial in informing future clinical best practices, while qualitative research could facilitate more in-depth exploration of the specific barriers faced by nonbinary youth. This information is essential for the design of inclusive systems of care and health policy. Research focused on understanding health care providers' beliefs, attitudes, and level of knowledge about non-binary youth can also inform development of educational resources and training opportunities. Research findings should be accessible so that non-binary youth and their families can access research results and information about the standards of care they are entitled to receive.

\section{Strengths and limitations}

This study is the first to report on disparities in health outcomes and health care access between non-binary youth and binary youth within a national health survey. One of the key limitations in this study is the non-probability sample design; however, given the population size and recruitment challenges, it may be impractical to design probability samples that will include enough trans and non-binary youth for separate analyses. Another limitation is the small number of trans-specific health care questions that were asked. In future research, we recommend addressing pubertal suppression, gender-affirming surgeries, non-binary youth's interactions with providers of gender-affirming care, impacts of misgendering, and satisfaction with care received, through both quantitative and qualitative methods. Finally, non-binary youth connected to trans communities or medical providers may have been over-represented, as recruitment methods focused on gender clinics and trans youth organizations.

\section{Conclusion}

Non-binary youth in Canada disproportionately experience poor health outcomes and barriers to care, as compared to their binary trans peers. Current standards of care address the needs of non-binary individuals as well as the role of primary care providers in delivering gender-affirming medical care. Additional research into best practices is warranted; however, there are many steps primary care providers can immediately take to increase capacity to provide gender-affirming care for non-binary youth.

\section{Declaration of conflict of interest/ethical approval}

The authors declare no conflicts of interest. Institutional ethics approval was obtained from the University of British Columbia (certificate \#H12-03129), University of Winnipeg (certificate \#GT856), and Dalhousie University (certificate \#2012-2804).

\section{Acknowledgements}

This research was supported by grant \#MOP 119472 from the Canadian Institutes of Health Research, Institute of Gender and Health. The funder had no involvement in study design; in the collection, analysis, and interpretation of data; in the writing of the report; or in the decision to submit the article for publication.

\section{Notes}

1. Only $p$ values .01 or smaller reported here to adjust for type 1 errors from the number of comparisons.

\section{References}

Austin, A. (2016). "There I am": A grounded theory study of young adults navigating a transgender or gender nonconforming identity within a context of oppression and invisibility. Sex Roles, 75(5-6), 215-230. doi:10.1007/s11199-016-0600-7

Barrett, J. (2016). Doctors are failing to help people with gender dysphoria. BMJ (Clinical Research Ed.), 352, i1694.

Bauer, G. R., Zong, X., Scheim, A. I., Hammond, R., \& Thind, A. (2015). Factors impacting transgender patients' discomfort with their family physicians: A respondent-driven sampling survey. PLOS One, 10(12), e0145046. doi:10.1371/journal.pone.0145046

Beemyn, G. (2015a). Coloring outside the lines of gender and sexuality: The struggle of nonbinary students to be recognized. The Educational Forum, 79(4), 359-361. doi:10.1080/00131725.2015.1069518

Beemyn, G. (2015b). Still waiting for an introduction to gender nonconformity: A review of transgender 101: A simple guide to a complex issue. Journal of LGBT Youth, 12(1), 87-89. doi:10.1080/19361653.2014.935549

Bosse, J. D., \& Chiodo, L. (2016). It's complicated: Gender and sexual orientation identity in LGBTQ youth. Journal of Clinical Nursing, 25(23-24), 3665-3675. doi:10.1111/jocn.13419

Browne, K. (2004). Genderism and the bathroom problem: (Re)materialising sexed sites, (re)creating sexed bodies. Gender, Place \& Culture, 11(3), 331-346. doi:10.1080/0966369042000258668

Coleman, E., Bockting, W., Botzer, M., Cohen-Kettenis, P., DeCuypere, G., Feldman, J., ... Zucker, K. (2012). Standards of care for the health of transsexual, transgender, and gender-nonconforming people, version $7 . \quad$ Retrieved from http://www.wpath.org/site_page.cfm?pk_association_webpage_men u D 1351\&pk_association_webpage D 3926

Connolly, M. D., Zervos, M. J., Barone, C. J. II., Johnson, C. C., \& Joseph, C. L. M. (2016). The mental health of transgender youth: Advances in understanding. Journal of Adolescent Health, 59(5), 489-495. doi:10.1016/j.jadohealth.2016.06.012

Conron, K. J., \& Scout Austin, S. B. (2008). "Everyone has a right to, like, check their box:" Findings on a measure of gender identity from a cognitive testing study with adolescents. Journal of LGBT Health Research, 4(1), 1-9. doi:10.1080/15574090802412572

Craig, S., Austin, A., Brennan, D. J., \& Alessi, E. J. (2015). A communitybased affirmative coping skills intervention for sexual and gender minority youth: Preliminary feasibility of project AFFIRM. Presented at the 2015 APHA Annual Meeting \& Expo (Oct. 31 - Nov. 4, 2015), APHA. Retrieved from https://apha.confex.com/apha/143am/webprogram/Paper336341.html

De Pedro, K. T., Gilreath, T. D., Jackson, C., \& Esqueda, M. C. (2017). Substance use among transgender students in California public middle and high schools. Journal of School Health, 87(5), 303-309. doi:10.1111/josh.12499

de Vries, A. L. C., McGuire, J. K., Steensma, T. D., Wagenaar, E. C. F., Doreleijers, T. A. H., \& Cohen-Kettenis, P. T. (2014). Young adult psychological outcome after puberty suppression and gender reassignment. Pediatrics, 134(4), 696-704. doi:10.1542/peds.20132958

Deutsch, M. (2016). Guidelines for the primary care and gender-affirming care of transgender and gender nonbinary people (2nd ed.). San Francisco, CA: Center of Excellence for Transgender Health.

Fausto-Sterling, A. (2000). The five sexes, revisited. The Sciences, 40(4), $18-23$.

Frohard_Dourlent, H., Dobson, S., Clark, B. A., Duoll, M., \& Saewyc, E. M. (2016). "I would have preferred more options": Accounting for non-binary youth in health research. Nursing Inquiry, 24(e12150), 19. doi:10.1111/nin. 12150

Gridley, S. J., Crouch, J. M., Evans, Y., Eng, W., Antoon, E., Lyapustina, M., \& Breland, D. J. (2016). Youth and caregiver perspectives on barriers to gender-affirming health care for transgender youth. Journal of Adolescent Health, 59(3), 254-261. doi:10.1016/j.jadohealth.2016.03.017

Halberstam, J. (1998). Female masculinity. Durham, NC: Duke University Press. 
Harrison, J., Grant, J., \& Herman, J. L. (2012). A gender not listed here: Genderqueers, Gender Rebels, and OtherWise in the National Transgender Discrimination Survey. LGBTQ Public Policy Journal at the Harvard Kennedy School, 2(1), 13-24. Retrieved from http://escholarship.org/uc/item/2zj46213

Hastings, J. (2017). Approach to genderqueer, gender non-conforming, and gender nonbinary people. Retrieved from http://transhealth.ucsf.edu/trans?pageDguidelinesgendernonconforming

Hidalgo, M. A., Ehrensaft, D., Tishelman, A. C., Clark, L. F., Garofalo, R., Rosenthal, S. M., \& Olson, J. (2013). The gender affirmative model: What we know and what we aim to learn. Human Development, 56(5), 285-290. doi:10.1159/000355235

Huft, M. (2008). Statistically speaking: The high rate of suicidality among transgender youth and access barriers to medical treatment in a society of gender dichotomy: Mental health, medicine, and the law. Children's Legal Rights Journal, 28, 53-56

Jones, A. (2016, December 19). Redefining gender. Retrieved from http://www.nationalgeographic.com/magazine/2017/01/exploregender-glossary-terminology/

Kenagy, G. P. (2005). Transgender health: Findings from two needs assessment studies in Philadelphia. Health and Social Work, 30(1), 19-26.

Kosenko, K., Rintamaki, L., Raney, S., \& Maness, K. (2013). Transgender patient perceptions of stigma in health care contexts. Medical Care, 51(9), 819-822. doi:10.1097/MLR.0b013e31829fa90d

LGBT Health Program. (2015). Guidelines and protocols for homone therapy and primary health care for trans clients. Toronto, ON: Sherbourne Health Centre.

Nicolazzo, Z. (2014). "Couldn't I be both Fred and Ginger?": Teaching about nonbinary identities through memoir. Journal of LGBT Youth, 11(2), 171-175. doi:10.1080/19361653.2013.878860

Olson, K. R., Durwood, L., DeMeules, M., \& McLaughlin, K. A. (2016). Mental health of transgender children who are supported in their identities. Pediatrics, 137(3), 1-8. doi:10.1542/peds.2015-3223

Olson-Kennedy, J., Cohen-Kettenis, P. T., Kreukels, B. P. C., MeyerBahlburg, H. F. L., Garofalo, R., Meyer, W., \& Rosenthal, S. M. (2016). Research priorities for gender nonconforming/transgender youth: Gender identity development and biopsychosocial outcomes. Current Opinion in Endocrinology \& Diabetes and Obesity, 23(2), 172-179. doi:10.1097/MED.0000000000000236

Poteat, T., German, D., \& Kerrigan, D. (2013). Managing uncertainty: A grounded theory of stigma in transgender health care encounters. Social Science \& Medicine, 84, 22-29. doi:10.1016/j.socscimed.2013.02.019

Rainbow Health Ontario, \& Speck, K. (2016). Primary Health Care for Trans Clients. Retrieved from https://www.rainbowhealthontario.ca/ TransHealthGuide/introterms.html

Rankin, S., \& Beemyn, G. (2012). Beyond a binary: The lives of gendernonconforming youth. About Campus, 17(4), 2-10. doi:10.1002/abc. 21086

Reisner, S. L., Bradford, J., Hopwood, R., Gonzalez, A., Makadon, H., Todisco, D., \& Mayer, K. (2015). Comprehensive transgender healthcare: The gender affirming clinical and public health model of Fenway health. Journal of Urban Health, 92(3), 584-592. doi:10.1007/s11524-015-9947-2

Reisner, S. L., Greytak, E. A., Parsons, J. T., \& Ybarra, M. L. (2015). Gender minority social stress in adolescence: Disparities in adolescent bullying and substance use by gender identity. Journal of Sex Research, 52(3), 243-256. doi:10.1080/00224499.2014.886321

Richards, C., Bouman, W. P., Seal, L., Barker, M. J., Nieder, T. O., \& T'Sjoen, G. (2016). Non-binary or genderqueer genders. International Review of Psychiatry, 28(1), 95-102. doi:10.3109/09540261.2015.1106446

Scelfo, J. (2015, February 3). A university recognizes a third gender: Neutral. The New York Times. Retrieved from https://www.nytimes.com/2015/02/08/education/edlife/auniversityrecognizes-a-third-gender-neutral.html

Scheim, A. I., \& Bauer, G. R. (2015). Sex and gender diversity among transgender persons in Ontario, Canada: Results from a respondent- driven sampling survey. Journal of Sex Research, 52(1), 1-14. doi:10.1080/00224499.2014.893553

Simons, L., Schrager, S. M., Clark, L. F., Belzer, M., \& Olson, J. (2013). Parental support and mental health among transgender adolescents. Journal of Adolescent Health, 53(6), 791-793. doi:10.1016/j.jadohealth.2013.07.019

Smith, A., Stewart, D., Poon, C., Peled, M., Saewyc, E., \& McCreary Centre Society. (2014). From Hastings Street to Haida Gwaii: Provincial results of the 2013 BC Adolescent Health Survey. Vancouver, BC: McCreary Centre Society. Retrieved from http://www.mcs.bc.ca/pdf/From_Hastings_ Street_To_Haida_Gwaii.pdf

Sperber, J., Landers, S., \& Lawrence, S. (2005). Access to health care for transgendered persons: Results of a needs assessment in Boston. International Journal of Transgenderism, 8(2-3), 75-91. doi:10.1300/J485v08n02_08

Travers, R., Bauer, G. R., Pyne, J., Bradley, K., Gale, L., \& Papadimitriou, M. (2012). Impacts of strong parental support for trans youth: A report prepared for Children's Aid Society of Toronto and Delisle Youth Services. Toronto, ON: TransPULSE. Retrieved from http://transpulseproject.ca/wp-content/uploads/2012/10/Impacts-ofStrong-Parental-Support-for-Trans-Youth-vFINAL.pdf

Vance, S. R., Halpern-Felsher, B. L., \& Rosenthal, S. M. (2015). Health care providers' comfort with and barriers to care of transgender youth. Journal of Adolescent Health, 56(2), 251-253. doi:10.1016/j.jadohealth.2014.11.002

Veale, J. F., Frohard-Dourlent, H., Dobson, S., \& Clark, B. \& Canadian Trans Youth Health Survey Research Group (2015) Being safe, being me: Results of the Canadian trans youth health survey. Vancouver, BC: Stigma and Resilience Among Vulnerable Youth Centre, School of Nursing, University of British Columbia. Retrieved from http://saravyc.sites.olt.ubc.ca/files/2015/05/SARAVYC_TransYouth-Health-Report_EN_Final_Print.pdf

Wolowic, J. M., Heston, L. V., Saewyc, E. M., Porta, C., \& Eisenberg, M. E. (2016). Chasing the rainbow: Lesbian, gay, bisexual, transgender and queer youth and pride semiotics.Culture, Health \& Sexuality. doi:10.1080/13691058.2016.1251613

\section{Appendix}

Survey questions and response options.

\section{Health outcomes}

\section{Mental health}

In general, how would you describe your mental health? [excellent; good; fair; poor]

\section{Self-injury}

During the past 12 months, how many times did you hurt or injure yourself on purpose without wanting to die? (For example, by cutting, burning, or bruising yourself on purpose). [0 times; 1 or 2 times; 3 - 5 times; $6-9$ times; 10 - 19 times; 20 or more times]

\section{Suicide}

Younger youth: During the past 12 months, did you ever seriously consider attempting suicide? [yes; no]

Older youth: Have you ever seriously considered committing suicide or taking your own life? [yes; no; don't know] [If yes:] Has this happened in the past 12 months? [yes; no; don't know]

\section{Attempted suicide past year}

Younger youth: During the past 12 months, how many times did you actually attempt suicide? [0 times; 1 time; 2 or 3 times; 4 or 5 times; 6 or more times]

Older youth: Have you ever attempted to commit suicide or tried taking your own life? Did this happen in the past 12 months? [yes; no; don't know] 


\section{Alcohol}

In the last 4 weeks how often did you drink alcohol (liquor, wine, beer, or coolers)? [once or twice; once or twice each week; 3 or 4 times each week; 5 or 6 times each week; once each day; more than once each day; did not drink alcohol in the last 4 weeks; never drank alcohol in lifetime]

\section{Marijuana}

Younger youth: In the last 12 months, how often did you use cannabis (also known as marijuana, "weed", "pot", "grass", hashish, "hash", hash oil, etc.)? [1 or 2 times; 3 to 5 times; 6 to 9 times; 10 to 19 times; 20 to 39 times; 40 or more times; used, but not in the last 12 months; never used in lifetime; don't know what cannabis is]

Older youth: Have you ever used [marijuana, cannabis or hashish] in the past 12 months? [yes; no; don't know]

\section{Cigarettes}

Younger youth: During the last 30 days, on how many days did you smoke a cigarette? [ 0 days; 1 or 2 days; $3-5$ days; $6-9$ days; 10-19 days; 20 - 29 days; all 30 days]

Older youth: In the past month, on how many days have you smoked 1 or more cigarettes? [open response]

\section{Health care access}

\section{Family doctor}

Do you have a regular family doctor? [yes; no]

\section{Walk-in clinics}

Do you use walk-in clinics as your primary source of health care? [yes; no]

Trans identity or experience

Does your current family doctor know about your trans identity or experience? [yes; no, not sure]

\section{Comfort}

[Youth with a family doctor:] How comfortable are you discussing your trans status and trans-specific health care needs with your family doctor? [very comfortable; comfortable; uncomfortable; very uncomfortable]

How comfortable would you be discussing your trans status and/or trans-related health care needs with a doctor you did not know? [very comfortable; comfortable; somewhat uncomfortable; not at all comfortable]

\section{Foregone care}

Younger youth: In the past 12 months, have you thought you needed medical help because you were physically sick or hurt, but didn't get it? [yes; no]

Younger youth: In the past 12 months, have you thought you needed emotional or mental health services, but didn't get them? [yes; no]

Older youth: During the past 12 months, was there ever a time when you felt that you needed health care but you didn't receive it? [yes; no; don't know]

\section{Hormone therapy}

Have you ever taken hormones for trans-related reasons? [yes; no]

[Youth who have ever taken hormones:] From which source(s) have you ever received your hormones? [family doctor or GP; specialist; internet pharmacy; friend or relative; street/strangers; herbals or supplements; veterinary sources; other(s), please specify]

If you have never taken hormones, which best describes your situation? [not planning on taking hormones; still deciding if taking hormones is right for me; can't find a doctor to prescribe hormones; other, please specify] 